\title{
GAMMA-RAY BURST OBSERVATIONS WITH ISON NETWORK
}

\author{
A. Pozanenko ${ }^{1}$, L. Elenin ${ }^{2}$, E. Litvinenko ${ }^{3}$, A. Volnova ${ }^{1,4}$, A. Erofeeva $^{5}$,

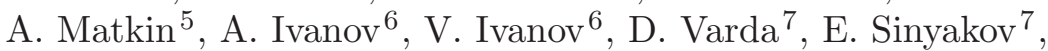 \\ V. Nevski ${ }^{8}$, Yu. Krugly ${ }^{9}$, A. Erofeev ${ }^{5}$, N. Tungalag ${ }^{10}$, R. Inasaridze ${ }^{11}$, \\ O. Kvaratskhelia ${ }^{11}$, V. Kouprianov ${ }^{3}$ and I. Molotov ${ }^{2}$
}

\begin{abstract}
We present details of the ISON network for GRB follow-up and complete list of observations in 2010-2012.
\end{abstract}

\section{The network description and results}

The International Scientific Optical Network (ISON) comprises several worldwide (Fig. 1) small aperture automated telescopes (Table 1). The ISON project is originally devoted to space debris observations (Molotov et al. 2008). Since 2010 ISON started observations of GRB. The shortest time delay after GRB trigger $(130 \mathrm{~s})$ was achieved in robotic mode of ISON-NM. After two years of GRB followup (Table 2) one can conclude that the network of small aperture telescopes is an efficient tool for GRB detection and photometry. Totally we observed 33 GRBs, detected 15 optical transients, and in several cases we succeed to build a dense light

\footnotetext{
1 Space Research Institute of the Russian Academy of Sciences, Moscow, Russia e-mail: apozanen@iki.rssi.ru

2 Institute for Applied Mathematics of the Russian Academy of Sciences, Moscow, Russia

3 Central Astronomical Observatory of the Russian Academy of Sciences, Pulkovo, Russia

4 Sternberg Astronomical Institute of Lomonosov Moscow State University, Moscow, Russia

${ }^{5}$ Ussuriysk Astrophysical Observatory of the Russian Academy of Sciences, Far East Branch, Gornotayojnoye, Russia

${ }^{6}$ Kuban State University, Krasnodar, Russia

7 Blagoveschensk Educational State University, Blagoveschensk, Russia

8 Astronomical Science Center "Proekt-Tekhnika", Moscow, Russia

${ }^{9}$ Institute of Astronomy of Kharkiv National University, Kharkiv, Ukraine

10 Research Center of Astronomy \& Geophysics of the Mongolian Academy of Sciences, Ulaanbaatar, Mongolia

11 Kharadze Abastumani Astrophysical Observatory of Ilia State University, Georgia
} 


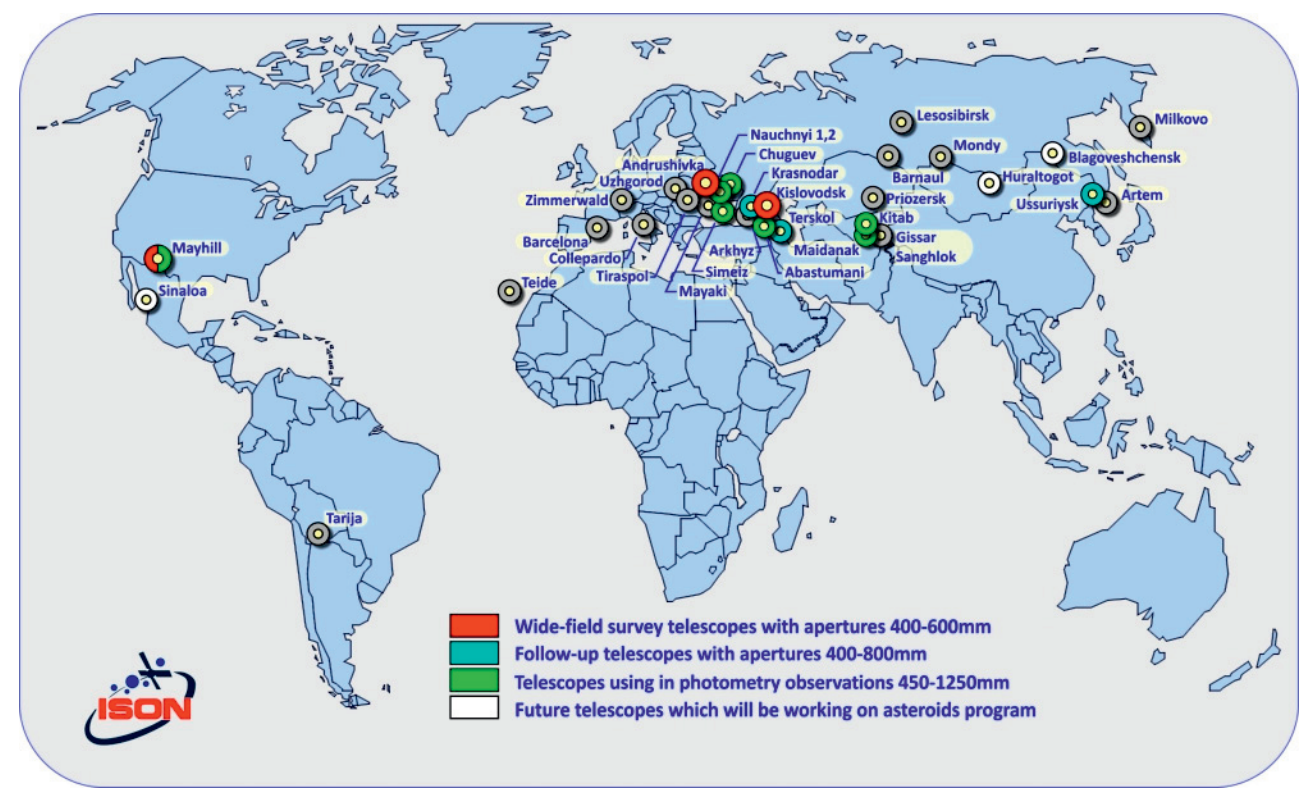

Fig. 1. Map of ISON observatories.

Table 1. ISON and cooperative telescopes used for GRB observations. * - taken from Kornilov et al. (2010), other data obtained from own monitoring.

\begin{tabular}{l|l|l|l}
\hline Site & Telescope & Aperture, m & $\begin{array}{l}\text { Num. of clear } \\
\text { night hours/year }\end{array}$ \\
\hline (M)ilkovo & ORI-22 & $0.22(\mathrm{f} / 2.45)$ & $\mathrm{n} / \mathrm{a}$ \\
(U)ssuriysk & VT-50 & $0.5(\mathrm{f} / 2.3)$ & 900 \\
(B)lagoveshchensk & ORI-22 & $0.22(\mathrm{f} / 2.45)$ & 1600 \\
(H)ureltogot & ORI-40 & $0.4(\mathrm{f} / 2.3)$ & 1400 \\
(K)itab & ORI-40 & $0.4(\mathrm{f} / 2.3)$ & 1650 \\
(S)anglokh & VT-78a & $0.19(\mathrm{f} / 1.54)$ & $\mathrm{n} / \mathrm{a}$ \\
(A)bastumani & AS-32 & $0.7(\mathrm{f} / 3)$ & 1200 \\
K(I)slovodsk & SANTEL-400A & $0.4(\mathrm{f} / 3)$ & $1343^{*}$ \\
K(R)asnodar & Astrosib RC-508 & $0.51(\mathrm{f} / 6.3)$ & 1200 \\
(N)ew Mexico & Centurion-18 & $0.45(\mathrm{f} / 2.8)$ & 1800 \\
\hline
\end{tabular}

curve of early optical afterglow (Fig. 2). Future development of GRB follow up within ISON includes robotization of the telescopes, elaboration of new wide-field telescopes for fast and deep follow up GBM/Fermi and synchronous observations of FOV of space borne observatories (e.g. Pozanenko et al. 2003), development of automatic pipelines for astrometry and photometry, and installation the telescopes in new observatories (Fig. 1). 
Table 2. GRBs observed with ISON and cooperative observatories. In the column (2) the time delay between GRB trigger and start of observation is presented. In the (3) we provide brightness of the OT at the first detection (or $3 \sigma \mathrm{UL}$ ). Most of observations are unfiltered. Brightness is estimated against USNO-B1.0 reference stars, $\mathrm{R}$ mag.

\begin{tabular}{l|c|l|l||l|l|l|l}
\hline GRB & Delay & $R_{m a g}$ (obs.) & GCN num. & GRB & Delay & $R_{m a g}($ obs. $)$ & GCN num. \\
\hline $100728 \mathrm{~B}$ & $16.4^{\mathrm{m}}$ & $18.36(\mathrm{~N})$ & 11012,11045 & $120402 \mathrm{~A}$ & $20.9^{\mathrm{m}}$ & $>19.8(\mathrm{~K})$ & 13200 \\
$101804 \mathrm{~A}$ & $3.27^{\mathrm{d}}$ & $19.79(\mathrm{~N})$ & 11129,11133 & $120404 \mathrm{~A}$ & $22.0^{\mathrm{m}}$ & $17.35(\mathrm{~B})$ & 13235 \\
$100901 \mathrm{~A}$ & $8^{\mathrm{m}}$ & $17.82(\mathrm{~N})$ & 11184,11234 & $120802 \mathrm{~A}$ & $12.5^{\mathrm{m}}$ & $>17.5(\mathrm{~N})$ & $13556,13609,13712$ \\
$100906 \mathrm{~A}$ & $13.5^{\mathrm{m}}$ & $15.89(\mathrm{U})$ & 11395 & $120803 \mathrm{~A}$ & $3.7^{\mathrm{m}}$ & $>17.3(\mathrm{~N})$ & 13617 \\
$110719 \mathrm{~A}$ & $1.05^{\mathrm{h}}$ & $19.70(\mathrm{~N})$ & 12177 & $120811 \mathrm{C}$ & $20.0^{\mathrm{m}}$ & $17.90(\mathrm{~K})$ & 13693,13679 \\
$110820 \mathrm{~A}$ & $15.4^{\mathrm{m}}$ & $>19.2(\mathrm{~K})$ & 12321 & $120816 \mathrm{~A}$ & $15^{\mathrm{m}}$ & $>18.5(\mathrm{R})$ & $\mathrm{n} / \mathrm{a}$ \\
$111016 \mathrm{~A}$ & $3.82^{\mathrm{h}}$ & $>19.2(\mathrm{~K})$ & 12486 & $120907 \mathrm{~A}$ & $12.9^{\mathrm{m}}$ & $18.55(\mathrm{I})$ & 13761 \\
$111029 \mathrm{~A}$ & $3.61^{\mathrm{m}}$ & $>18.3(\mathrm{~N})$ & 12500 & $120911 \mathrm{~A}$ & $2.45^{\mathrm{m}}$ & $>18.4(\mathrm{~N})$ & 13759 \\
$111205 \mathrm{~A}$ & $2.90^{\mathrm{d}}$ & $>19.8(\mathrm{~N})$ & 12736 & $120923 \mathrm{~A}$ & $6.43^{\mathrm{m}}$ & $>19.7(\mathrm{~N})$ & 13820 \\
$111228 \mathrm{~A}$ & $0.84^{\mathrm{d}}$ & $19.27(\mathrm{~N})$ & 12832 & $121001 \mathrm{~A}$ & $7.1^{\mathrm{m}}$ & $19.0(\mathrm{R})$ & $\mathrm{n} / \mathrm{a}$ \\
$120106 \mathrm{~A}$ & $4.81^{\mathrm{h}}$ & $>18.5(\mathrm{~K})$ & 12830 & $121011 \mathrm{~A}$ & $6.2^{\mathrm{m}}$ & $16.46(\mathrm{U})$ & 13884 \\
$120116 \mathrm{~A}$ & $21.3^{\mathrm{m}}$ & $>19.4(\mathrm{~K})$ & 12899 & $121108 \mathrm{~A}$ & $9.0^{\mathrm{m}}$ & $\mathrm{n} / \mathrm{a}(\mathrm{S})$ & $\mathrm{n} / \mathrm{a}$ \\
$120118 \mathrm{~B}$ & $45.0^{\mathrm{m}}$ & $>19.5(\mathrm{~K})$ & 12900 & $121117 \mathrm{~A}$ & $1.75^{\mathrm{h}}$ & $>18.9(\mathrm{~B})$ & 13978 \\
$120119 \mathrm{~A}$ & $1.26^{\mathrm{h}}$ & $18.97(\mathrm{~N})$ & 12871,12881 & $121123 \mathrm{~A}$ & $5.2^{\mathrm{h}}$ & $19.01(\mathrm{~A})$ & 13988,14200 \\
$120121 \mathrm{~A}$ & $2.78^{\mathrm{h}}$ & $>19.7(\mathrm{~N})$ & 12887 & $121128 \mathrm{~A}$ & $0.43^{\mathrm{d}}$ & $20.24(\mathrm{~A})$ & 14201 \\
$120308 \mathrm{~A}$ & $3.3^{\mathrm{m}}$ & $17.30(\mathrm{~N})$ & 13019 & $121212 \mathrm{~A}$ & $2.3^{\mathrm{m}}$ & $20.7(\mathrm{~S})$ & 14071 \\
$120320 \mathrm{~A}$ & $12.6^{\mathrm{m}}$ & $>16.7(\mathrm{~B})$ & 13198 & & & & \\
\hline
\end{tabular}
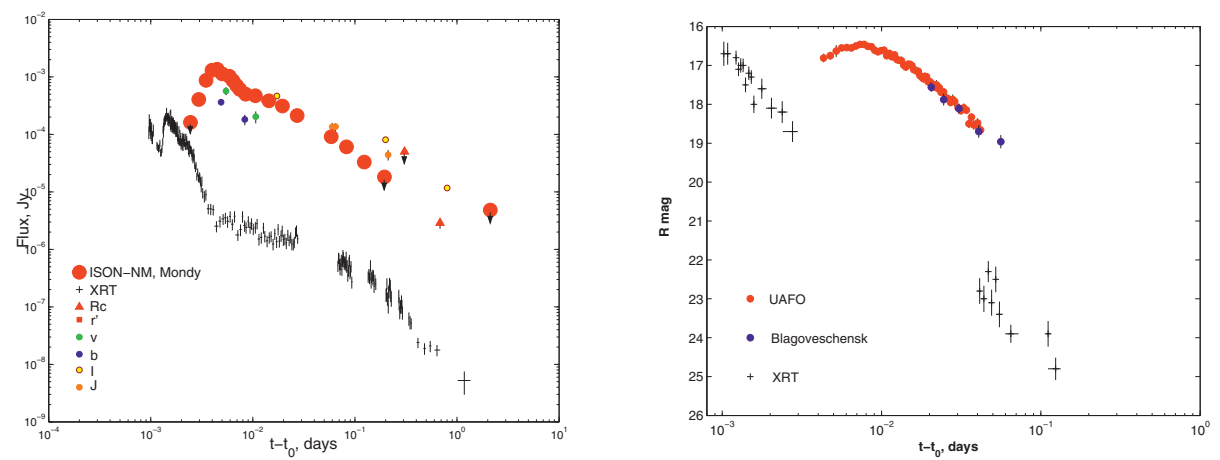

Fig. 2. Left: GRB 120308A: the ISON-MN began the observations of the optical transient 3.3 minutes after GRB trigger and continued it 5 hours. Right: GRB 121011A: the optical transient was recorded 6.2 minutes after the GRB trigger by Ussuriysk observatory.

The work was partially supported by RFBR grants 12-02-01336-a, 11-01-92202_Mong-a.

\section{References}

Molotov, I., Agapov, V., Titenko, V., et al., 2008, AdSpR, 41, 1022

Kornilov, V., Shatsky, N., Voziakova, O., et al., 2010, MNRAS, 408, 1233

Pozanenko, A., Chernenko, A., Beskin, G., et al., 2003, ASPC, 295, 457 
